\title{
$\Omega$
}

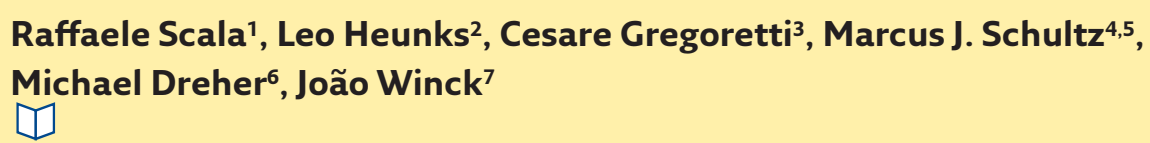

'Pulmonlogy and RICU, ASL 8 Arezzo Respiratory Intensive Care Unit, S. Donato Hospital, Arezzo, Italy. ${ }^{2}$ Dept of Intensive Care Medicine, VU University Medical Centre, Amsterdam, The Netherlands. ${ }^{3}$ Dept of Biopathology and Medical Biotechnologies (DIBIMED), Section of Anesthesia, Analgesia, Intensive Care and Emergency, Policlinico P. Giaccone, University of Palermo, Palermo, Italy. ${ }^{4}$ Dept of Intensive Care and Laboratory of Experimental Intensive Care and Anesthesiology, Academic Medical Center, Amsterdam, The Netherlands. ${ }^{5}$ Mahidol Oxford Tropical Medicine Research Unit, Bangkok, Thailand. ${ }^{6}$ Division of Pneumology, University Hospital RWTH, Aachen, Germany. ${ }^{7}$ Pulmonology, Faculdade de Medicina do Porto, Porto, Portugal.

\section{Assembly 2: respiratory intensive care}

\section{Meet the Assemblies}

Cite as: Scala R, Heunks L, Gregoretti C, et al. Assembly 2: respiratory intensive care. Breathe 2017; 13: 147-148.
Assembly 2, the home of groups "02.01: acute critical care" and "02.02: noninvasive ventilatory support", is a growing and active assembly within the European Respiratory Society (ERS). The number of members in Assembly 2 is steadily increasing, reaching a total of 846 in 2016 . The members of Assembly 2 are young, with up to $40 \%$ of the members of Assembly 2 aged $<40$ years, and scientifically active, with 120 accepted abstracts at the recent ERS International Congress 2106 in London. The overarching aims of Assembly 2 are to promote respiratory intensive care within ERS through educational activities (in conjunction with HERMES (Harmonised Education in Respiratory Medicine for European Specialists)) and hands-on courses, to facilitate science within the field of respiratory intensive care, to disseminate the latest scientific novelties, to stimulate interactions among ERS members with interest in respiratory intensive care and to attract new ERS members.

Assembly 2 is heterogeneous, multidisciplinary and multiprofessional; incorporating physicians with diverse educational backgrounds, such as pulmonology, anaesthesiology, internal medicine, intensive care medicine and emergency medicine, and allied healthcare professions, such as nurses, physiotherapists and physiologists. All members share dedication and enthusiasm for respiratory intensive care in its broadest context.

\section{Group 02.01: acute critical care}

Group 02.01 is dedicated to the broad area of intensive care medicine, focusing on pathophysiology of and treatment strategies for acute respiratory failure. Many of its members are involved in research in this field, including specific topics like (lung-protective) mechanical ventilation and weaning from ventilatory support, but also sepsis, diaphragm dysfunctions, tracheostomy care, end-of-life care and health economics related to intensive care medicine. More recently, the group has started to cover the topic of "difficult airway management in critically ill patients" through a skills workshop, and "lung imaging in critically ill patients" through an ERS task force. This group invests in activities together with Assembly 1: Clinic; Assembly 4: Clinical Physiology, Sleep and Pulmonary Circulation; Assembly 7: Paediatrics; Assembly 9: Allied Respiratory Professionals; and Assembly 10: Respiratory Infections.

\section{Group 02.02: noninvasive ventilatory support}

Group 02.01 has a specific interest in both acute and chronic noninvasive ventilation (NIV) for several 
disorders, including neuromuscular or restrictive diseases, chronic obstructive pulmonary disease, acute and chronic heart failure and obesity hypoventilation syndromes.

NIV is one of the most relevant developments of respiratory medicine in the last years. Although advanced knowledge has been extensively made on NIV, there are still some areas to progress and innovate concerning home mechanical ventilation, NIV titration, set up and monitoring. The group is working to propose consensus statements and recommendations that will be endorsed by ERS in the forthcoming years. On a regular basis, the group organises several postgraduate courses like "NIV: basic concepts" and a "NIV advanced course".

The more recent course, called "Comprehensive management of ventilator-dependent patients", was held in Barcelona October 6-8, 2016 and was very successful with 70 participants from all over the world (www.facebook.com/EuropeanRespiratorySociety/ posts/10154496436600119)

Finally, a new ERS skill courses on NIV and Respiratory Intermediate Care Units will also be planned in the future.

\section{Conflict of interest}

Disclosures can be found alongside this article at breathe.ersjournals.com 